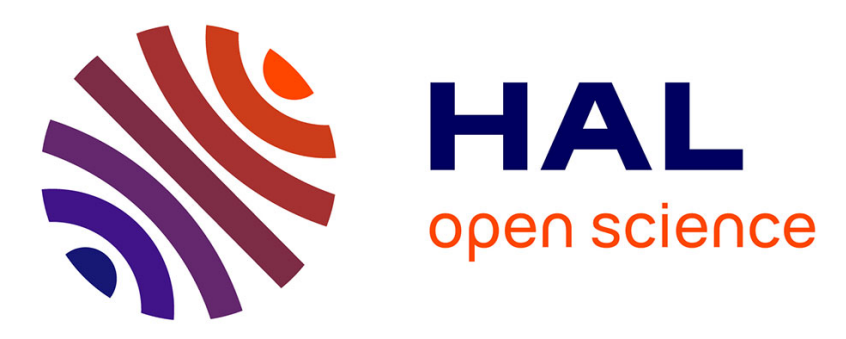

\title{
Comparison of Visual Registration Approaches of 3D Models for Orthodontics
}

Raphaël Destrez, Benjamin Albouy-Kissi, Sylvie Treuillet, Yves Lucas, Arnaud Marchadier

\section{- To cite this version:}

Raphaël Destrez, Benjamin Albouy-Kissi, Sylvie Treuillet, Yves Lucas, Arnaud Marchadier. Comparison of Visual Registration Approaches of 3D Models for Orthodontics. Advances Concepts for Intelligent Vision Systems, Aug 2011, Belgium. pp.647-657, 10.1007/978-3-642-23687-7_58 . hal00648845

\section{HAL Id: hal-00648845 \\ https://hal.science/hal-00648845}

Submitted on 6 Dec 2011

HAL is a multi-disciplinary open access archive for the deposit and dissemination of scientific research documents, whether they are published or not. The documents may come from teaching and research institutions in France or abroad, or from public or private research centers.
L'archive ouverte pluridisciplinaire HAL, est destinée au dépôt et à la diffusion de documents scientifiques de niveau recherche, publiés ou non, émanant des établissements d'enseignement et de recherche français ou étrangers, des laboratoires publics ou privés. 


\title{
Comparison of Visual Registration Approaches of 3D Models for Orthodontics
}

\author{
R. Destrez ${ }^{1,3}$, B. Albouy-Kissi ${ }^{2}$, S. Treuillet ${ }^{1}$, Y. Lucas $^{1}$, A. Marchadier ${ }^{1,3}$ \\ ${ }^{1}$ Laboratoire PRISME, Polytech’Orléans 12 rue de Blois, 45067 Orléans, France \\ ${ }^{2}$ Université d'Auvergne, ISIT, BP 10448, 63000 Clermont-Ferrand, France \\ ${ }^{3}$ UsefulProgress, 23 rue d'Anjou, 75008 Paris, France \\ \{raphael.destrez, arnaud.marchadier\}@gmail.com \\ sylvie.treuillet@univ-orleans.fr \\ yves.lucas@bourges.univ-orleans.fr \\ benjamin.albouy@iut.u-clermont1.fr
}

\begin{abstract}
We propose to apply vision techniques to develop a main tool for orthodontics: the virtual occlusion of two dental casts. For that purpose, we process photos of the patient mouth and match points between these photos and the dental 3D models. From a set of 2D/3D matches of the two arcades, we calculate the projection matrix, before the mandible registration under the maxillary through a rigid transformation. We perform the mandible registration minimizing the reprojection errors. Two computation methods, depending on the knowledge of camera intrinsic parameters, are compared. Tests are carried out both on virtual and real images. In the virtual case, assumed as perfect, we evaluate the robustness against noise and the increase of performance using several views. Projection matrices and registration efficiency are evaluated respectively by reprojection errors and the differences between the rigid transformation and the reference pose, recorded on the six degrees of freedom.
\end{abstract}

Keywords: Visual registration, 3D models, projection matrix, orthodontics

\section{Introduction}

Orthodontics is a dental specialty concerned with the correction of bad positions of jaws and teeth to optimize dental occlusion for functional and esthetical purposes. The first classification of malocclusions was established by E.H Angle in 1898[1]. Today, the treatment can involve a chirurgical intervention or dental device implantation. Commonly, the orthodontist uses dental casts of patient's arcades to plan the treatment and manually achieve an attempt of occlusion. As in many other medical 
applications, this manual practice can be replaced by new planning and diagnostic imaging tools. Dental casts can be completed by dentition photos [2] or radiographies [3]. Other imaging tools can be used like CT-scan [4][5] or face scan devices [6][7][8][9] that provide additional information about soft tissues and face appearance, but still requiring dental casting. Anyway these techniques are not widespread because there are expensive and bulky.

A real contribution of imaging is the possibility to create 3D numerical models of

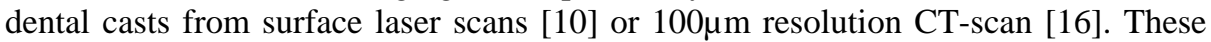
techniques differ on speed and cost but none enables an adjustment of the dental arcades corresponding to the real occlusion of patient.

This information can be obtained with a silicone device used during the teeth printing [11] or another reference [7] but it requires additional operation for the practitioner.

CT-scan or heavy devices [12] can be used to obtain the pose of the mandible relative to the maxillary but these techniques are not widespread. In practice, after the scan of dental casts, the search for the optimal occlusion is computerized [16]. A technician moves manually the mandible to modify the occlusion of the dental arcades 3D models. For this task, he relies on dedicated software and photos of mouth patient (Fig. 2a above). This process is very tedious and the result is operator dependent.

So, our goal is to perform automatically most of the numerical occlusion setting. Thereby, it will be faster and independent of the operator. We chose to use real photos of the dentition to capture the natural occlusion of the arcades and to map it on the virtual model. This strategy avoids adding another step to the current orthodontist practice. In this paper, we carry out a comparative study of two methods to evaluate the relevance and precision of this approach. These studies are carried out for different configurations of viewpoints and 2D/3D correspondences.

\section{Model Registration from Mouth Images}

The 3D models of the two dental arcades are obtained separately, preventing any expression in a shared work coordinate system with the original fitting of the arcades. Consequently, it is necessary to determine a rigid transformation setting in occlusion the mandible against the maxillary.

We propose to use colors photos of mouth patient that display the real "in vivo" occlusion for several viewpoints (Fig. 2a). We choose a set of correspondences between 3D points on the surface model and their 2D projections in images to estimate this rigid transformation. The projection matrix obtained from homogenous coordinates can be expressed by the relation between 3D point, $\boldsymbol{M}_{j}$, and its projection in the image $i, \boldsymbol{m}_{i j}$ :

$$
\boldsymbol{m}_{i j} \propto \boldsymbol{P}_{i} \boldsymbol{M}_{j}
$$

where $\propto$ means «by a scaling factor». The $3 \times 4$ projection matrix $\boldsymbol{P}_{i}$ can be decomposed into a product of two matrices. 
These contain intrinsic parameters of the camera and extrinsic parameters:

$$
\boldsymbol{P}_{i}=\boldsymbol{K}\left[\boldsymbol{R}_{i} \mid \boldsymbol{t}_{i}\right] .
$$

where $\boldsymbol{t}_{i}$ and $\boldsymbol{R}_{i}$ are respectively the translation and the rotation defining the pose of the camera and $\boldsymbol{K}$ is the camera calibration matrix where scorresponds to the pixel slope, $x_{0}$ and $y_{0}$ are the frame principal point coordinates, $r_{x}$ and $r_{y}$ horizontal and vertical sensor resolutions and $f$ is the focal camera distance:

$$
\boldsymbol{K}=\left(\begin{array}{ccc}
f r_{x} & s & x_{0} \\
0 & f r_{y} & y_{0} \\
0 & 0 & 1
\end{array}\right)
$$

For current cameras, pixels are commonly considered as squares and the principal point at the center of pictures, leading to the relations $r_{x}=r_{y}=r,\left(x_{0}, y_{0}\right)=\left(x_{p}, y_{p}\right)$ with little impact on the precision. Moreover, it allows the calculation of the matrix $\mathrm{K}$ from the picture size and the focal distance $f r$ given in pixels.

From a set of 2D/3D correspondences for the two arcades, we propose a two step resolution of the problem: firstly we estimate the projection matrix for each view from maxillary correspondences, before the registration of the mandible with analytical (DeMenthon) [13] or minimization [14] methods. Two situations can be encountered, depending on the knowledge of the focal distance. If the camera is calibrated, we must only determinate its pose (position and orientation), embedding six degrees of freedom (dof in the following). Conversely, if we don't know the focal length, we calculate the complete $\boldsymbol{P}_{i}$ projection matrix with 11 dof. In addition, the rigid registration of the mandible provides 6 dof of more. Several views can be used jointly during the registration.

\subsection{Estimation of $P_{i}$ With $K$ Knowledge (WiK)}

In this method called «WiK», the determination of the projection matrix of the image $i$ amounts to the extrinsic parameters estimation: three Euler's angles defining the orientation $\boldsymbol{R}_{i}$ and three translation components of the $\boldsymbol{t}_{i}$ vector. Firstly, we use the POSIT algorithm of DeMenthon [13] for a rough estimation of the six parameters, from correspondences established on the maxillary. Then, from this initialization, the six parameters are optimized by the Levenberg-Marquardt algorithm minimizing the sum of the squared reprojection error:

$$
s e_{i}=\sum_{j=1}^{n_{i}} \sum_{k=1}^{2}\left[m_{i j k}-\widetilde{m}_{i j k}\right]^{2} .
$$

where $\mathrm{i}, \mathrm{j}$, and $\mathrm{k}$ are respectively picture, point and coordinate indexes, $m_{i j \mathrm{k}}$ refers to a reference point coordinate in the picture and $\widetilde{m}_{i j k}$ refers to a the reprojection of the corresponding 3D point by the $\boldsymbol{P}_{i}$ matrix. 
For evaluation in experiments presented in section 3, we use the reprojection mean squared error (MSE):

$$
m s e_{i}=\sqrt{\frac{1}{n_{i}} \sum_{j=1}^{n_{i}} \sum_{k=1}^{2}\left[m_{i j k}-\widetilde{m}_{i j k}\right]^{2}}
$$

\subsection{Etimation of $P_{i}$ Without $K$ Knowledge (WoK)}

When $\boldsymbol{K}$ is unknown, the projection matrix $\boldsymbol{P}_{i}$ is estimated by least square minimization on the maxillary 2D/3D correspondences. The last element of $\boldsymbol{P}_{i}$ is fixed to 1 . Then, the 11 parameters of the $\boldsymbol{P}_{i}$ matrix are optimized with the LevenbergMarquardt algorithm by minimizing $s e_{i}$ (equation 4). This approach is called «WoK».

\subsection{Registration of the Mandible}

One projection matrix estimated, the mandible is registrated under the maxillary in occlusion position. The reference coordinate system of the maxillary is illustrated figure 1: the origin is the gravity center of the point cloud of the maxillary, the $\mathrm{Y}$ axis is facing up and the $\mathrm{Z}$ axis is parallel to the rows of molars. The reference coordinate system of the mandible reference is similar but placed on the gravity center of the mandible point cloud.

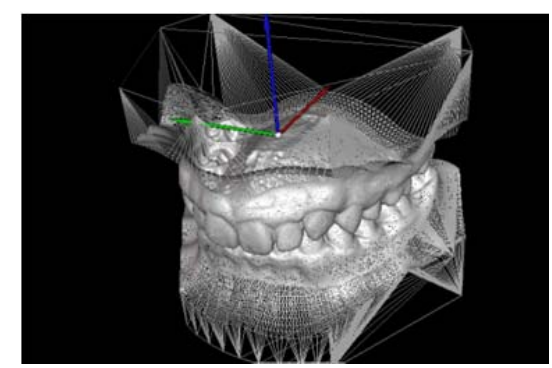

Fig. 1. Reference coordinate system of the registration.

The registration is carried out by calculating the rigid transformation $\boldsymbol{T}$ according to the fact that 3D point projections of the mandible are superimposed with the projections selected in the pictures. Projections are obtained using the matrix $\boldsymbol{P}_{i}$ estimated from the maxillary points where a 3D point $\boldsymbol{M}_{\text {mand }}$ on the mandible and its projection $\boldsymbol{m}_{i, \text { mand }}$ on the photo $i$ is linked by:

$$
\boldsymbol{m}_{i, \text { mand }} \propto \boldsymbol{P}_{i} \boldsymbol{T} \boldsymbol{M}_{\text {mand }} .
$$


The transformation $\boldsymbol{T}$ embeds 6 dof: three Euler's angles and three translations estimated by minimizing the reprojection error (equation 4) using LevenbergMarquardt algorithm with an identity transform as initial conditions.

\section{Experiments}

To validate the two previous steps, tests have been carried out both on virtual and real images. From a "perfect" virtual case, we evaluate the robustness against noise and the increase of performance using several views. The virtual pictures are obtained by screenshots from the VTK rendering of 3D models (Fig. 2a). So, several points of view can be simulated controlling the acquisition settings (focal, view angle, etc...). Such virtual pictures are distortion free but we can raise and control the noise level on the point coordinates to simulate real watching process. Real pictures are taken with a numerical 8 mega pixels camera (Canon EOS 350D), a $60 \mathrm{~mm}$ lens and an annular flash (Fig. 2a). We use OpenCV for the calculation functions like POSIT [13] and the levmar functions [15] for the implementation of the Levenberg-Marquardt algorithm.

a)
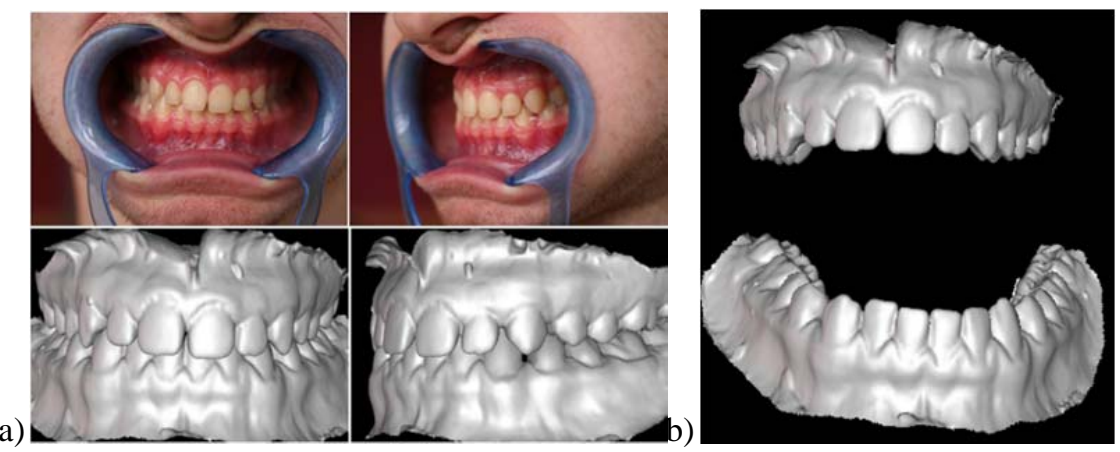

Fig. 2.(a)Front view (left) and side view (right)

(top) real image $(1300 \times 867)($ down $)$ virtual views $(1300 \times 867)$.

(b) Initial location of the 3D models before the registration.

\subsection{Projection Matrix Estimation}

To evaluate the estimation of projection matrix, the tests are carried out on 3D virtual models registrated in a good occlusion position decided by orthodontics specialist. Ten features are manually pointed on the 3D models of the maxillary, and ten others on the mandible one. The two previous methods are compared on virtual data and real pictures. Estimated projection matrices are evaluated with the reprojection errors (equation 5). We have done this test for two different points of view: a front view and a side view. This second point of view is one of the most lateral views that we can obtain. The figure 2a represents a typical configuration of this point of view. 


\subsubsection{Virtual Data}

While perfect projections are simulated by using the VTK screen, 2D/3D correspondences can be considered as perfect (Fig. 3). From this "perfect" case, we add an uniform noise on the 2D point coordinates to compare the robustness of WiK and WoK methods against noise. The tables 1 and 2 present the average \pm the standard deviation, and the maximal values of the reprojection error (equation 5) respectively for maxillary and mandible points, after the optimization step. For each noise level, we average a hundred random realizations. To evaluate the error introduced by a human operator, we also test a manual 2D point picking.

Regarding the mandible reprojection errors, we can observe that the WiK method surpasses globally the WoK method for the estimation of the projection matrix. It remains that the non-perfect estimation of $\mathrm{K}$ used in the WiK method could explain the opposite observation for the maxillary errors. As only 2D/3D maxillary correspondences are used to estimate the projection matrix, the maxillary errors are lower than mandible errors. The errors increase linearly with the noise level (from zero in the perfect case). It should be noticed that these errors remain small compared to the size of pictures expressed in pixels. Concerning the manual selection, mandible MSE for the side view reaches 21.6 pixels $(0.93 \mathrm{~mm})$ for the WoK method while it is 10.3 pixels for the WiK method. The manual picking errors are equivalent to a noise around 6 pixels. This demonstrates that capturing manually the $2 \mathrm{D}$ reprojection with a high precision is really a tricky task.

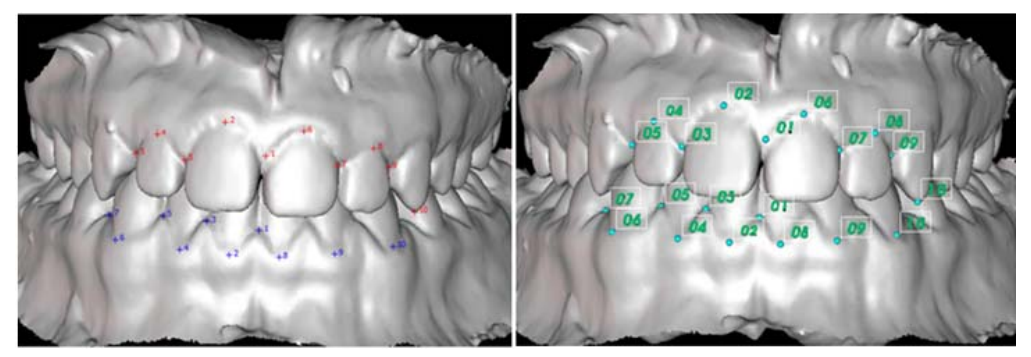

Fig. 3. (left) Virtual 2D picture obtained by 3D model projection on the screen with 3D points projections (right) dentition 3D model with manually selected 3D points. 
Table 1. Reprojection MSE (in pixels) for the WiK method and two virtual viewpoints.

\begin{tabular}{|c|c|c|c|c|}
\hline \multirow{2}{*}{$\begin{array}{c}\text { Noise } \\
\text { (pixels) }\end{array}$} & $\begin{array}{c}\text { MSE } \\
\text { maxillary }\end{array}$ & $\begin{array}{c}\text { Max. error } \\
\text { maxillary }\end{array}$ & $\begin{array}{c}\text { MSE } \\
\text { mandible }\end{array}$ & $\begin{array}{c}\text { Max. error } \\
\text { mandible }\end{array}$ \\
\hline 2 & $1.37 \pm 0.19$ & 2.17 & $1.92 \pm 0.31$ & 3.06 \\
\hline 4 & $2.73 \pm 0.39$ & 4.34 & $3.83 \pm 0.61$ & 6.12 \\
\hline Manual & $3.48 \pm 1.53$ & 5.81 & $5.66 \pm 2.96$ & 10.7 \\
\hline & \multicolumn{5}{|c|}{ Side view } \\
\hline 2 & $1.36 \pm 0.18$ & 2.18 & $1.98 \pm 0.32$ & 3.17 \\
\hline 4 & $2.72 \pm 0.35$ & 4.37 & $3.96 \pm 0.63$ & 6.34 \\
\hline Manual & $4.00 \pm 1.93$ & 7.50 & $6.35 \pm 2.86$ & 10.3 \\
\hline
\end{tabular}

Table 2. Reprojection MSE (in pixels) for the WoK method and two virtual viewpoints.

\begin{tabular}{|c|c|c|c|c|}
\hline \multirow{2}{*}{$\begin{array}{c}\text { Noise } \\
\text { (pixels) }\end{array}$} & $\begin{array}{c}\text { MSE } \\
\text { maxillary }\end{array}$ & $\begin{array}{c}\text { Max. error } \\
\text { maxillary }\end{array}$ & $\begin{array}{c}\text { MSE } \\
\text { mandible }\end{array}$ & $\begin{array}{c}\text { Max. error } \\
\text { mandible }\end{array}$ \\
\hline 2 & $1.08 \pm 0.22$ & 1.81 & $5.22 \pm 2.16$ & 8.45 \\
\hline 4 & $2.16 \pm 0.44$ & 3.61 & $10.45 \pm 4.36$ & 16.94 \\
\hline Manual & $2.90 \pm 1.36$ & 5.20 & $14.8 \pm 4.90$ & 22.1 \\
\hline & \multicolumn{5}{|c|}{ Side view } \\
\hline 2 & $1.09 \pm 0.20$ & 1.92 & $3.69 \pm 1.30$ & 6.04 \\
\hline 4 & $2.18 \pm 0.39$ & 3.84 & $7.37 \pm 2.62$ & 12.09 \\
\hline Manual & $3.00 \pm 1.57$ & 6.14 & $12.9 \pm 6.00$ & 21.6 \\
\hline
\end{tabular}

\subsubsection{Real Case}

The estimation of the projection matrix has been tested on two real images (Fig.2a) with a manual point selection. Five tests have been carried out to display a tendency. In table 3, we can observe, that mandible reprojection errors are still more important than maxillary ones. In this case, $1 \mathrm{~mm}$ corresponds to 11,5 pixels. We note again the superiority of the WiK method. Especially in the manual case, we can see that reprojection errors are higher than on virtual images for the WoK method. This can be explained by a higher complexity for the manual picking in the real case, while the color picture had not the same appearance (colors, texture, different lightings...) than the 3D model (shadows, relief...). In the virtual case, the simulated images are really similar to the 3D model so that ease the selection of correspondences. 
Table 3. Reprojection MSE (in pixels) for the WiK and WoK methods on two real pictures.

\begin{tabular}{|c|c|c|c|c|}
\hline \multirow{2}{*}{ Method } & \multicolumn{4}{|c|}{ Front view } \\
\cline { 2 - 5 } & $\begin{array}{c}\text { MSE on the } \\
\text { maxillary }\end{array}$ & $\begin{array}{c}\text { Max. error } \\
\text { maxillary }\end{array}$ & $\begin{array}{c}\text { MSE on the } \\
\text { mandible }\end{array}$ & $\begin{array}{c}\text { Max.error } \\
\text { mandible }\end{array}$ \\
\hline WiK & $5.24 \pm 2.72$ & 9.57 & $8.38 \pm 2.90$ & 13.6 \\
\hline WoK & $3.77 \pm 1.29$ & 5.93 & $36.6 \pm 11.1$ & 53.5 \\
\hline & \multicolumn{5}{|c|}{ Side view } \\
\hline WiK & $5.44 \pm 2.68$ & 10.6 & $7.70 \pm 3.08$ & 12.2 \\
\hline WoK & $4.54 \pm 2.19$ & 8.70 & $16.8 \pm 4.50$ & 24.0 \\
\hline
\end{tabular}

\subsection{Mandible Registration}

To test the registration both for virtual and real images, the 3D models of arcades are positioned in the same initial configuration, corresponding to a practical configuration. The registration has been done separately with each viewpoint and then using both images together. From the reference pose, the rigid transformation between mandible and maxillary is defined by the Euler's angles $\alpha=-15^{\circ}, \beta=-6^{\circ}$ and $\gamma=6^{\circ}$ and by the translation components $\mathrm{X}=-5 \mathrm{~mm}, \mathrm{Y}=-24 \mathrm{~mm}$ and $\mathrm{Z}=-11 \mathrm{~mm}$. This configuration is presented in the figure $2 \mathrm{~b}$. The quality of the final registration is evaluated by the differences on the six parameters of the rigid transformation. The reference pose is the one obtained by the orthodontist expert with the current technique. We present only the best results, obtained for a registration involving two viewpoints together. It includes also the reprojection error occurring after the registration step because this is the actual criteria minimized by the LM algorithm during the registration. As previously, a uniform noise is added on the perfect data to evaluate algorithm robustness and the errors are averaged on 100 random tests. Moreover, we carry out a set of manual selection tests. Currently, in orthodontic practice, there are no quantitative criteria to estimate the quality of a registration. We considered that a position error of $0.1 \mathrm{~mm}$ and an orientation error of $0.5 \mathrm{~mm}$ are relevant bounds compared to teeth dimensions.

\subsubsection{Virtual Data}

Concerning the reprojection errors observed after the registration, they are lower than in table 3. In the manual selection case with the WiK method using only one picture, reprojection errors are 3.24 pixels for the front view and 3.80 for the side view. In the same configuration, for the WoK method, errors are 4.50 pixels for the front view and 4.65 for the side view. We can explain that by the fact that the minimized criterion is the mandible reprojection error.

Moreover, the errors are slightly lower if the registration is done with one view rather than with two (in the same configuration for a registration using two points of view, 
reprojection error is 4.27 pixels for the front view). In this latter case, optimization is a compromise between the two sets of points. This difference is more important for the WoK method.

The figures 4, 5 and table 4 present the 3D differences after the registration relative to the reference positioning provided by the orthodontist expert. Globally, the errors with the WiK method are less important than with the WoK one with a linear dependence to noise. In the "perfect" case, the registration using two viewpoints provide very good results. In the graphs, horizontal straight lines correspond to the manual selection case (MS).

Firstly, we have examined the positioning of the gravity center (Fig. 4 and table 4). The $\mathrm{Z}$ translation is the less precise because this direction corresponds to the depth in the pictures. The Y translation is the more precise because this axis is perpendicular to the camera axis. For the WiK method, this tendency is not respected, but all errors are low. For the manual selection the maximal error is $0.17 \mathrm{~mm}$. For the WoK method, this tendency is respected but errors can be more important with a maximum of $0.38 \mathrm{~mm}$ for the manual selection.

Secondly, we focused on the angle errors (Fig. 5 and table 4). The $\beta$ and $\alpha$ angles seem to be the most difficult to evaluate with respectively $0.48^{\circ}$ and $0.33^{\circ}$ errors for the manual selection with the WiK method. Whatever the method, the $\gamma$ angle is the easiest to estimate. Another time, WoK method has higher errors than WiK method.

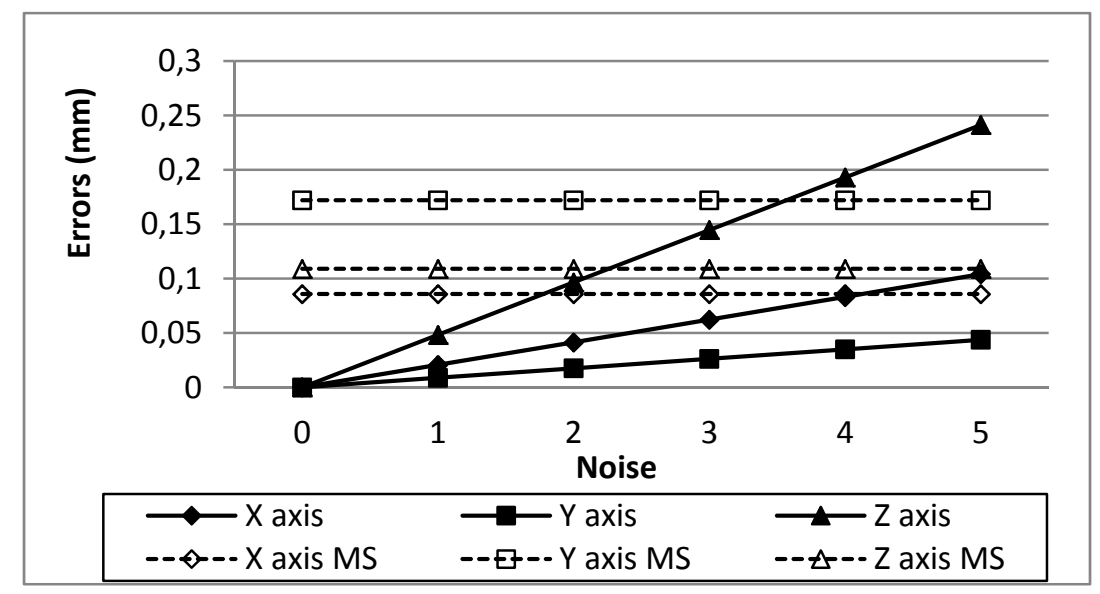

Fig. 4. Gravity center errors on the virtual data (WiK method). 


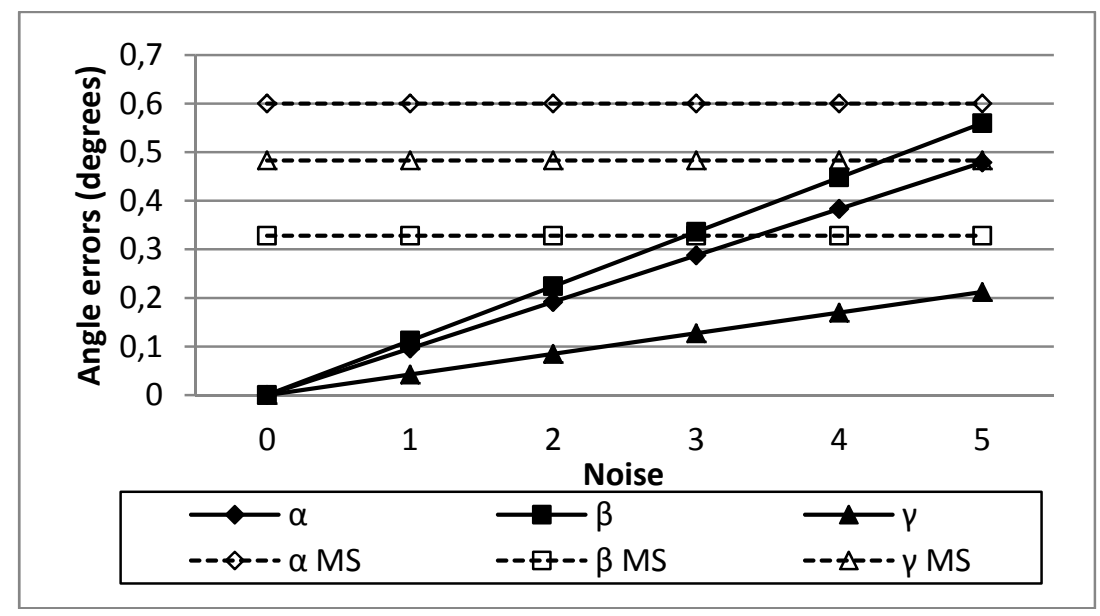

Fig. 5. Angle errors on the virtual data (WiK method).

Table 4. 3D errors on the virtual data for the WoK method (in pixels for the gravity center errors and in degrees for the angle errors).

\begin{tabular}{|c|c|c|c|c|}
\hline Gravity center errors & $\mathrm{X}$ & $\mathrm{Y}$ & $\mathrm{Z}$ & $3 \mathrm{D}$ \\
\hline 2 & $0.10 \pm 0.068$ & $0,08 \pm 0,064$ & $0,16 \pm 0,116$ & 0.21 \\
\hline MS & 0,37 & 0,23 & 0,38 & 0,57 \\
\hline Angles errors & $\alpha$ & $\beta$ & $\gamma$ \\
\hline 2 & $0,43 \pm 0,31$ & $0,42 \pm 0,32$ & $0,26 \pm 0,17$ \\
\hline MS & 1.36 & 0,75 & 0,32 \\
\hline
\end{tabular}

\subsubsection{Real Case}

For the 3D registration errors (table 5),WiK method produces again globally better results than WoK method. The errors are not very high, but they cannot always afford the precision required for a real dental occlusion. The tests carried out with a unique view reveal sometimes some aberrations. In that case, the registration is not realistic and some 3D errors can be high (until $\mathrm{Z}=73 \mathrm{~mm}$ for the WoK method). By combining the two viewpoints, the registration is clearly improved and generally the aberrations almost disappear. Nevertheless, 3D errors are higher than with the virtual case. In average, the translation error is maintained below $0.8 \mathrm{~mm}$ and the rotation error below 1.2 degrees for the WiK method (respectively $2 \mathrm{~mm}$ and $4^{\circ}$ for the WoK method).

As in the virtual case, we observe an increase of the reprojection error by using the both picture. Nevertheless, these errors are higher than for the virtual case and the WiK method is still more effective (average of 6.86 pixels) against the WoK method (average of 12.7 pixels).

Repetitive tests on real images show that registration is very sensitive to the point selection even if we try to click carefully the same features. Sometimes, when the 
registration is moderate with one view and sharp with the other one, the quality of the registration combining the two views is tempered by the poor results with the first view.

Table 5. 3D errors relative to the translation $(\mathrm{mm})$ and rotation (degrees) after registration in the real case.

\begin{tabular}{|c|c|c|c|c|}
\hline & $\mathrm{X}$ & $\mathrm{Y}$ & $\mathrm{Z}$ & $3 \mathrm{D}$ \\
\hline WiK & $0.27 \pm 0.21$ & $0.56 \pm 0.22$ & $0.14 \pm 0.16$ & $0.68 \pm 0.2$ \\
\hline WoK & $0.94 \pm 0.46$ & $1.27 \pm 0.63$ & $0.58 \pm 0.42$ & $1.81 \pm 0.5$ \\
\hline & $\alpha$ & \multicolumn{2}{|c|}{$\beta$} & $\gamma$ \\
\hline WiK & $1.26 \pm 0.86$ & $0.95 \pm 0.743$ & $0.27 \pm 0.20$ \\
\hline WoK & $1.20 \pm 1.00$ & $3.74 \pm 0.77$ & $1.77 \pm 1.07$ \\
\hline
\end{tabular}

\section{Conclusion}

These first experiments demonstrate that the automatic registration of two dental arcades can be achieved from images of mouth patient. The proposed methods are effective in the " perfect » virtual case, both for the projection matrix estimation and model registration. Nevertheless, the performance of these tests decreases when adding noise, uncertainties or with real shot constrains. It will be interesting to complete this study observing the impact of distortions on virtual cases. Globally, WiK method provides the better results than WoK method for all tested aspects even if in the manual cases, reprojection errors and 3D errors remain significant after registration. For the registration, reprojection error doesn't seem the unique and the best criteria to minimize: relative distance between several critical points or 3D distances obtained from the calculation of triangulated 3D points could be more relevant.

In the future, the most challenging problem is to realize an automatic matching between singular points or lines on 3D models of dental arcades and their corresponding features in the color pictures of the mouth. Our strategy will consist in bringing together the 2D and 3D representations to simplify the matching process. For this goal, we will carry out a 3D reconstruction from two or more views to extract and describe 3D features (bending radius) or reciprocally take advantage of 2D features (colorimetric gradient, texture). Moreover, we will enhance the 3D model with texture mapping.

\section{Acknowledgements}

We thank the Ortho-Concept laboratory (http://www.ortho-concept.com/) and more especially Daniel Julie for providing us most of numerical data. 


\section{References}

1. Angel, E.H.: Treatment of malocclusion of the teeth and fractures of the maxillae: Angle's system. S.S. White Dental Manufacturing Company, 6th edition (1900)

2. Ettorre, G. et al.: Standards for digital photography in cranio-maxillo-facial surgery - Part I: Basic views and guidelines. Journal of Cranio-Maxillofacial Surgery 34, pp. 65-73 (2006)

3. Broadbent, B. Holly: A new X-ray technique and its application to orthodontia. The Angle Orthodontics, vol. 1, n. 2, pp. 45--66 (April 1931)

4. Chung, R., Lagravere, M., Flores-Mir, C., Heo, G., Carey, J. P., Major, P.: Analyse comparative des valeurs céphalométriques de céphalogrammes latéraux générés par CBCT versus céphalogrammes latéraux conventionnels. International Orthodontics, vol. 7, nº 4, pp. 308--321, (Dec. 2009)

5. Plooij, J.M. et al.: Digital three-dimensional image fusion processes for planning and evaluating orthodontics and orthognathic surgery. A systematic review. Int Journal of Oral and Maxillofacial Surgery. In Press.

6. Terajima, M. et al.: A 3-dimensional method for analyzing facial soft-tissue morphology of patients with jaw deformities. American Journal of Orthodontics and Dentofacial Orthopedics 135, pp. 715--722 (2009)

7. Sohmura, Taiji et al.: High-resolution 3-D shape integration of dentition and face measured by new laser scanner. New York, USA : Institute of Electrical and Electronics Engineers, vol. 23. (2004)

8. Schendel, S.A. \& Lane, C.: 3D Orthognathic Surgery Simulation Using Image Fusion. Seminars in Orthodontics 15, pp. 48--56 (2009)

9. Rangel, F.A. et al.: Integration of digital dental casts in 3-dimensional facial photographs. American Journal of Orthodontics and Dentofacial Orthopedics 134, pp. 820--826 (2008)

10. Asquith, J., Gillgrass, T., Mossey, P.:Three-dimensional imaging of orthodontic models: a pilot study. European Journal of Orthodontics, vol. 29, $\mathrm{n}^{\circ}$. 5, pp. 517-522 (Oct. 2007)

11. Alfano, S.G., Leupold, R.J.: Using the neutral zone to obtain maxillomandibular relationship records for complete denture patients. The Journal of Prosthetic Dentistry, vol. 85, n. 6, pp. 621--623 (June 2001)

12. Enciso, R., Memon, A., Fidaleo, D.A., Neumann, U., Mah, J.: The virtual craniofacial patient: 3D jaw modeling and animation. Studies in Health Technology and Informatics, vol. 94, 65--71 (2003)

13. DeMenthon, D.F., Davis, L.S.: Model-based object pose in 25 lines of code. Int. Journal of Computer Vision 15, pp. 123--141 (1995)

14. Marquardt, D.: An algorithm for least-squares estimation of nonlinear parameters. SIAM Journal on Applied Mathematics 11, pp. 431--441 (1963)

15. Lourakis, M.:levmar: Levenberg-Marquardt nonlinear least squares algorithms in $\{C\} /\{C\}++$. http://www.ics.forth.gr/ lourakis/levmar/ (Juil-2004)

16. http:// www.ortho-concept3d.com 